\title{
HEREDITARY SPHEROCYTOSIS PRESENTING IN CHILDHOOD IN AN ASIAN CHILDREN'S HOSPITAL
}

\section{Siok Hoon Ang ${ }^{1}$, Joyce Lam Ching $\mathrm{Mei}^{2}$}

Department of Paediatric Medicine, KK Women's and Children's Hospital (KKH), Singapore, siokhoon.ang@mohh.com.s@

2 Paediatric Haematology \& Oncology Service, KK Women's and Children's Hospital (KKH), Singapore, joyce.lam.c.m@singhealth.com.sg

\section{Introduction}

Hereditary spherocytosis (HS) is a red cell membrane disorder affecting membrane proteins $-\alpha$ and $\beta$ spectrin, ankyrin, band 3 or protein $4.2^{1}$. It is the most common cause of haemolytic anaemia in individuals of Northern European ancestry with a prevalence of 1 in $2000^{1,2}$. Although there are cases reported worldwide in all racial and ethnic groups, it is less common in South-East Asia (SEA). The heterogeneity of the condition results in individuals with differing severity varying from symptom-free carriers to severe haemolysis. To date, no cohort study on paediatric patients from Singapore with a predominantly Asian population has been conducted.

\section{Aim}

This study highlights the clinical features and complications of paediatric HS patients in our institution.

\section{Methods}

Clinical characteristics and complications of patients were obtained retrospectively from medical records of paediatric patients diagnosed with HS from 2006 - 2016 in KK Women's and Children's Hospital (KKH). Diagnosis was confirmed by osmotic fragility (OF) or eosin-5-maleimide (EMA) binding tests. Follow-up duration ranged from 4 months to 25 years (mean 7.75 years).

\section{Results}

Clinical characteristics of the 31 patients identified are shown in Table 1. Their presenting symptoms and complications are shown in Table 2 and 3 respectively.

Table 1:
Gender

Race

Mean age of diagnosis

Positive family history

Available genetic profiles

Haemoglobin $(\mathrm{Hb})$ level at diagnosis (g/dL)

No. of patients

Female: 21 (67.7\%)

Male: $10(32.3 \%)$

Chinese: $20(64.5 \%)$

Malays: 9 (29\%)

Others: 2 (6.5\%)

3.5 years (birth to 14.3 years)

- $12(38.7 \%)$ patients presented before 1 month of age ( 8 with neonatal jaundice (NNJ) requiring intense phototherapy or exchange transfusion, 1 with prolonged NNJ, 1 with NNJ screened due to family history, 2 with anaemia) 16 (52\%)

$3(9.7 \%)$

2 with SPTB gene mutation which codes for $\beta$ spectrin

1 with SLC4A1 gene mutation which codes for Band 3 protein

$<6: \quad 7(22.6 \%)$

6-7.9: $5(16.1 \%)$

8-9.9: $\quad 6(19.4 \%)$

10-11.9: $5(16.1 \%)$

$>12: \quad 8(25.8 \%)$

Mean Hb: 9.3 (4 - 16.5)

Table 2

Presenting symptoms

No. of patients

Jaundice

Pallor or anaemia

Incidental blood film finding

$15(48.4 \%)$

$14(45.2 \%)$

$4(12.9 \%)$

$2(6.5 \%)$
Table 3: Complications

Transfusion

$22(71 \%)$ required at least one transfusion. $9(29 \%)$ required frequent $(>4)$ transfusions

Splenectomy $6(19.4 \%)$

- Mean age: 12.5 years (7- 19 years)

- Reasons: 4 due to frequent transfusions, 1 due to concurrent cholecystectomy and $\mathbf{1}$ due to hypersplenism

Gallstones

$10(32.3 \%)$

- 3 required surgical removal

Haemolytic crisis

$22(71 \%)$

$8(25.8 \%)$ had parvovirus infection

Aplastic crisis

$3(9.7 \%)$

- 2 (6.5\%) had parvovirus infection

\section{Discussion}

The incidence of HS in our institution is low with only 31 patients identified over 10 years. This does not represent the true population incidence as the study was conducted on paediatric patients in a single institution, hence patients with milder phenotype who present in adulthood will not be represented. Mean age of diagnosis was 3.5 years, with 12 patients $(38.7 \%)$ diagnosed before 1 month of age. Eight patients had NNJ requiring intensive treatment, suggesting that severe jaundice should prompt an investigation into haemolytic conditions such as HS. This is similar to western studies where half of infants with HS developed severe NNJ compared to $8 \%$ of normal newborns ${ }^{3}$. Positive family history was present in at least half $(52 \%)$ of the patients, less than the $75 \%$ reported in western studies ${ }^{2}$. This figure may change with increased awareness and screening of siblings of affected patients.

Confirmatory studies with OF or EMA were conducted for all patients, in contrast to recommendation stating that confirmatory test is not necessary for patients with family history and typical clinical and laboratory features ${ }^{1,4}$. Genetic studies are reserved mainly for patients with severe phenotype requiring multiple blood transfusions at an early age. The 3 patients with genotyping done were infants aged 9 months to 3 years. Understanding their genotype can aid in the prognosis of the child as well as genetic counselling for their parents.

Patients generally present with jaundice and anaemia as the first signs of the condition. This is similar to western studies in children where anaemia was the most frequent complaint (50\%), followed by splenomegaly, jaundice and positive family history (all 10-15\%) ${ }^{3}$. Data on presence of splenomegaly was not captured in our study.

A significant percentage $(71 \%)$ of patients required at least one transfusion, with lowest $\mathrm{Hb}$ recorded between $2.6-6.8$. This is close to recommended practice to transfuse patients when $\mathrm{Hb}$ falls below $5-6^{3,4}$. Splenectomy was performed on 6 patients, with frequent transfusions being the most common reason. According to guidelines, splenectomy is indicated in patients with moderate to severe HS who require frequent transfusions or those with complications such as gallstones or growth failure ${ }^{3,4}$. Splenectomy was delayed till after 6 years old in all patients as recommended ${ }^{4}$. No partial splenectomy was done in our institution. Amongst the 3 patients requiring surgical intervention for gallstones, 2 were due to symptomatic gallstones while one was due to concurrent splenectomy. The practice of cholecystectomy in patients with asymptomatic gallstones going for splenectomy is still controversial ${ }^{4}$. Similar to other studies, most patients $(71 \%)$ experienced at least one haemolytic crisis, often triggered by viral infections ${ }^{1,3}$, of which, 8 had parvovirus infection, 3 had aplastic crisis.

\section{Conclusion}

Most paediatric patients in our institution are diagnosed early, with the majority requiring at least one transfusion. HS should be considered in neonates with early jaundice requiring intensive therapy, especially those with family history Collaborative research with other institutions would give better overview of HS incidence in Singapore.

\section{References}

1. Silverio P, Patrick G G, Narra M. Hereditary Spherocytosis. Lancet 2008; 372:1411-26

2. Paula H.B Bolton-Magg. Hereditary Spherocytosis; new guidelines. Arch Dis Child. 2004 Sep; 89(9): 809-812

3. Stefan E and Samuel EL. Hereditary Spherocytosis - Defects in Proteins that connect the membrane skeleton to the lipid bilayer. Semin Hematol 41:118-1412004

4. Paula H.B Bolton-Maggs, Jacob CL, Achille I et al. Guidelines for the diagnosis and management of hereditary spherocytosis - 2011 update 